\title{
Assessing the Integrated Community-Based Health Systems Strengthening initiative in northern Togo: a pragmatic effectiveness- implementation study protocol
}

\author{
Molly E. Lauria ${ }^{1 *}$ D, Kevin P. Fiori ${ }^{1,2,3}$, Heidi E. Jones ${ }^{4}$, Sesso Gbeleou ${ }^{5}$, Komlan Kenkou ${ }^{5}$, Sibabe Agoro ${ }^{6}$,
} Abdourahmane Diparidé Agbèrè ${ }^{7,8}$, Kelly D. Lue ${ }^{1}$ and Lisa R. Hirschhorn ${ }^{1,9}$

\begin{abstract}
Background: Over the past decade, prevalence of maternal and child morbidity and mortality in Togo, particularly in the northern regions, has remained high despite global progress. The causes of under-five child mortality in Togo are diseases with effective and low-cost prevention and/or treatment strategies, including malaria, acute lower respiratory infections, and diarrheal diseases. While Togo has a national strategy for implementing the integrated management of childhood illness (IMCl) guidelines, including a policy on integrated community case management (iCCM), challenges in implementation and low public sector health service utilization persist. There are critical gaps to access and quality of community health systems throughout the country. An integrated facility- and community-based initiative, the Integrated Community-Based Health Systems Strengthening (ICBHSS) initiative, seeks to address these gaps while strengthening the public sector health system in northern Togo. This study aims to evaluate the effect and implementation strategy of the ICBHSS initiative over 48 months in the catchment areas of 21 public sector health facilities.
\end{abstract}

Methods: The ICBHSS model comprises a bundle of evidence-based interventions targeting children under five, women of reproductive age, and people living with HIV through (1) community engagement and feedback; (2) elimination of point-of-care costs; (3) proactive community-based IMCl using community health workers (CHWs) with additional services including family planning, HIV testing, and referrals; (4) clinical mentoring and enhanced supervision; and (5) improved supply chain management and facility structures. Using a pragmatic type II hybrid effectiveness-implementation study, we will evaluate the ICBHSS initiative with two primary aims: (1) determine effectiveness through changes in under-five mortality rates and (2) assess the implementation strategy through measures of reach, adoption, implementation, and maintenance. We will conduct a mixed-methods assessment using the RE-AIM (reach, effectiveness, adoption, implementation, maintenance) framework. This assessment consists of four components: (1) a stepped-wedge cluster randomized control trial using a community-based household survey, (2) annual health facility assessments, (3) key informant interviews, and (4) costing and return-oninvestment assessments for each randomized cluster.

Discussion: Our research is expected to contribute to continuous quality improvement initiatives, optimize implementation factors, provide knowledge regarding health service delivery, and accelerate health systems improvements in Togo and more broadly.

\footnotetext{
* Correspondence: mlauria@integratehealth.org

'Community Health Systems Lab, Integrate Health/Santé Intégrée, Kara, Togo

Full list of author information is available at the end of the article
}

(c) The Author(s). 2019 Open Access This article is distributed under the terms of the Creative Commons Attribution 4.0 International License (http://creativecommons.org/licenses/by/4.0/), which permits unrestricted use, distribution, and reproduction in any medium, provided you give appropriate credit to the original author(s) and the source, provide a link to the Creative Commons license, and indicate if changes were made. The Creative Commons Public Domain Dedication waiver (http://creativecommons.org/publicdomain/zero/1.0/) applies to the data made available in this article, unless otherwise stated. 
(Continued from previous page)

Trial registration: ClinicalTrials.gov, NCT03694366, registered 3 October 2018

Keywords: Reproductive, Maternal, Child Health, RE-AIM, Health systems, Community health workers, Supportive supervision, $\mathrm{IMCl}$, iCCM, Togo

\section{Contributions to the literature}

- This protocol demonstrates how to utilize implementation science methodologies to conduct a pragmatic trial in a low-income setting in order to improve service delivery and apply findings into practice.

- It provides a model for embedded implementation research in public sector service delivery to facilitate demand-driven research and adoption of scientific findings into policy implementation. Close collaboration and ownership amongst practitioners, policymakers, and researchers are crucial to address research questions for policy change of empirical value to local communities.

- This study will evaluate the effectiveness and implementation strategy of an integrated facility- and community-based initiative within a low-income health system and aims to provide generalizable evidence to policymakers to inform national community health strategy decisions.

\section{Background}

While Togo has observed reductions in child mortality over the last few decades, accelerated progress is needed to achieve Sustainable Development Goal (SDG) goal 3.2, to reduce neonatal mortality to 12 per 1000 live births and under-five mortality to 25 per 1000 live births [1, 2]. Most recent subnational estimates from 2013-2014 show that the infant mortality rate in the northern region of Kara is 62 per 1000 live births and the under-five mortality rate is 130 per 1000 live births, compared to national rates of 49 and 88 per 1000 live births, respectively [3]. The principal causes of under-five deaths in Togo are diseases with effective and low-cost interventions and treatments, including malaria, acute lower respiratory infections, and diarrheal diseases [3]. Despite Togo's national plan for the integrated management of childhood illness (IMCI), a policy on integrated community case management (iCCM), and the Expanded Program on Immunization (EPI), Togo has not observed comparable reductions in child mortality as compared to neighboring peer nations including Burkina Faso, Ghana, and Guinea [4]. Furthermore, Togo's Ministry of Health $(\mathrm{MoH})$ reports that national utilization rates of public sector health facilities are less than $30 \%$, even though $62 \%$ of the population lives within $5 \mathrm{~km}$ of these facilities $[5,6]$.

\section{Context and Integrated Community-Based Health Systems Strengthening model}

Integrate Health $(\mathrm{IH})$ is a non-governmental organization (NGO) working in collaboration since 2004 with the Togolese $\mathrm{MoH}$ and community-based organizations (CBOs). This public-private partnership focuses on integrating community and public sector health services in the Kara region, initially focusing on $\operatorname{HIV}[7,8]$ and subsequently expanding to primary care $[9,10]$. In pursuit of an overarching research objective to improve community health systems through scientific methods, IH formed the Community Health Systems Lab (CHSL), which embeds implementation research and dissemination into $\mathrm{IH}$ operations including the current study protocol. See Fig. 1 for CHSL organizational details. IH aims to foster a learning health system approach, through locally produced evidence to both strengthen the effectiveness of our primary partner, the Togolese $\mathrm{MoH}$, and contribute to knowledge generation and uptake by dissemination with local, national, and global colleagues.

In 2014, IH developed a new partnership with the $\mathrm{MoH}$ to improve primary healthcare services by addressing the leading causes of premature mortality in the catchment areas of four public sector clinics in the Kozah district of the northern Kara region. These preliminary integrated facility- and community-based health interventions are referred to as the Integrated Community-Based Health Systems Strengthening (ICBHSS) model [11]. This model includes a bundle of evidence-based interventions adapted for implementation in Togo and is consistent with global best practices including the recent World Health Organization (WHO) community health worker (CHW) guideline [12]. The target population includes children under five, pregnant/post-partum women, women of reproductive age for family planning services, and people living with HIV infections. As detailed in Table 1, the ICBHSS model consists of: (1) community engagement and feedback [13-15]; (2) elimination of point-of-care costs for the target population [16-19]; (3) proactive community-based IMCI using salaried CHWs with additional 


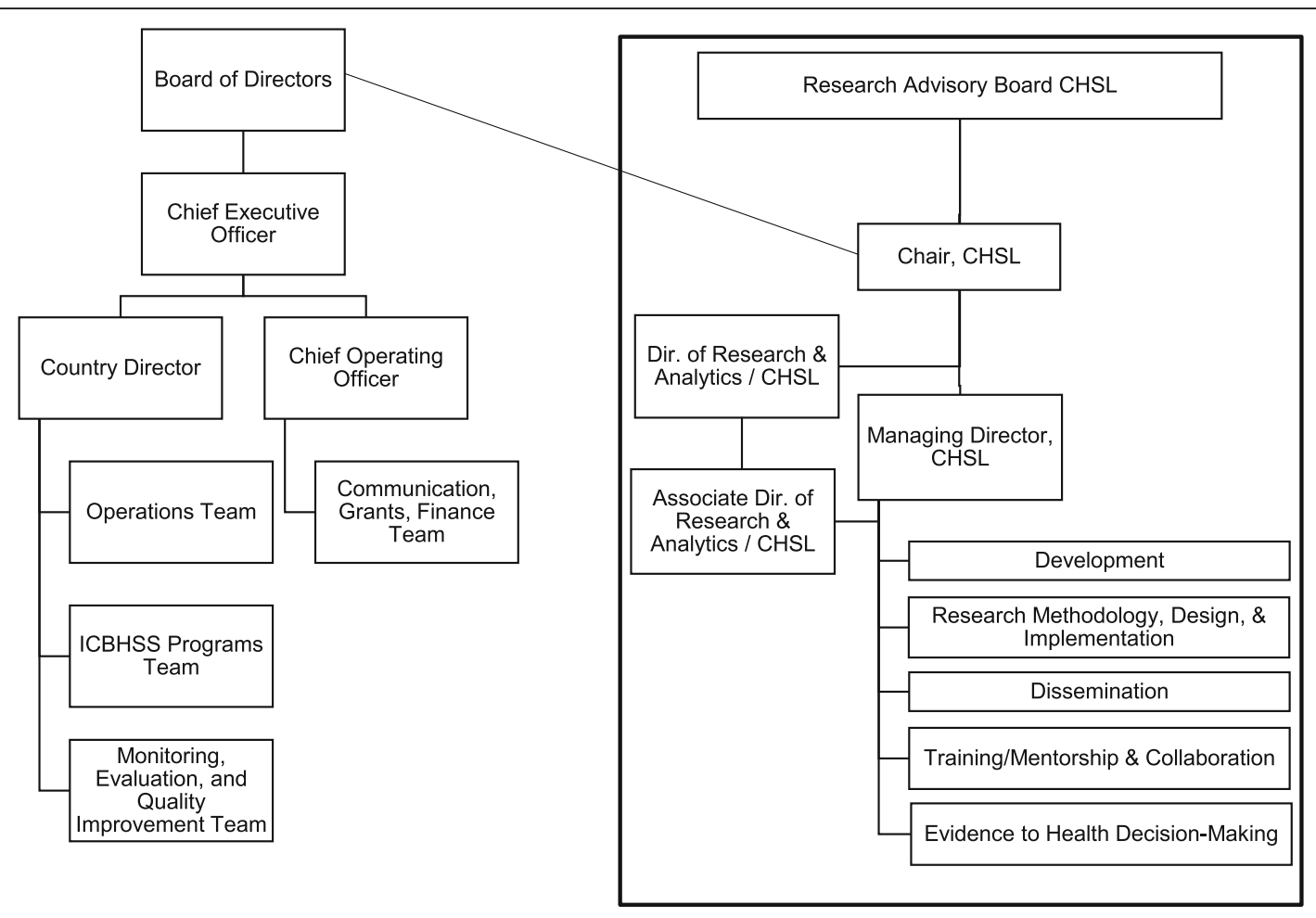

Fig. 1 Community Health Systems Lab (CHSL) organization structure within Integrate Health (IH) organizational chart

services including family planning and counseling, HIV testing, and referrals [11, 19-27]; (4) clinical mentoring and enhanced supervision at health centers [28-30]; and (5) facility and supply chain management [15, 31-33].

Preliminary results from the ICBHSS implementation pilot in the Kozah district observed a decline in underfive mortality and increased health service utilization for child and maternal health services at all four intervention sites (ClinicalTrials.gov Identifier: NCT03773913). This evaluation was conducted using a repeated, population-representative, cross-sectional household survey. We anticipate results from 48 months of implementation in 2019.

Following this successful preliminary pilot phase and considering the need, $\mathrm{IH}$ was requested by the $\mathrm{MoH}$ to expand the ICBHSS model to additional sites starting in 2018. In collaboration with the $\mathrm{MoH}$ and technical partners, IH is replicating the ICBHSS model in 21 distinct rural health facilities in four additional districts. In collaboration with $\mathrm{MoH}$ partners, IH's ICBHSS program team designed an implementation strategy for this expansion to enhance adoption, implementation, and sustainability of the model [34-36]. This strategy enables a rollout to a new district every 12 months, and is based on pilot experience, local contextual factors as well as budgetary and feasibility considerations. As part of this expansion launched in 2018, $\mathrm{IH}$ and $\mathrm{MoH}$ partners designed a stepped-wedge randomized trial to enable a rigorous assessment of intervention effectiveness and implementation strategies to inform national policy.

\section{Rationale for study design}

We selected a pragmatic type II hybrid effectivenessimplementation study, as it allows simultaneous mixedmethods assessments of intervention effectiveness and implementation strategies in "real life" health systems settings [35, 37]. Through this convergent design [38], we intend to routinely disseminate [39] our research findings to $\mathrm{MoH}$ partners. Effectiveness measures will provide evidence as to whether the ICBHSS intervention impacts child mortality and, via this pragmatic design, will provide more generalizable estimates than traditional study designs [35]. Assessing the implementation strategy will generate knowledge about process outcomes and feasibility including barriers and enabling factors, core components which are generalizable and where local adaptation is needed for replication in other settings. This actionable knowledge is a critical need for implementors in low- and middle-income settings, particularly for complex health systems interventions [12, 40].

Although our preliminary Kozah pilot study provided initial data to suggest ICBHSS's effectiveness, these results have limited capacity to establish causation due to single-arm design and lack of a valid comparison group. Furthermore, our pilot study was not designed to evaluate implementation strategies required to provide 
Table 1 Overview of Integrated Community-Based Health Systems Strengthening (ICBHSS) model components

\begin{tabular}{l} 
ICBHSS model component \\
\hline 1. Community engagement meetings and feedback \\
Evidence: [13-15] \\
2. Removal of point-of-care costs in IH intervention public sector health \\
facilities \\
Evidence: [16-19]
\end{tabular}

3. Proactive community-based IMCI using trained, equipped, supervised, and salaried CHWs with additional services including linkage to family planning and counseling, HIV testing, and referrals Evidence: [11, 19-27]

\section{Details}

- Pre-intervention consultation meetings with community leaders.

- Community participation in CHW selection.

- Biannual community review meetings with local leaders, community members, facility, and IH staff.

- IH staff presentation of programmatic results and updates.

- Community-provided feedback on ICBHSS implementation challenges, successes, and areas for improvement.

- Pertains to all children under five, pregnant/post-partum women, women of reproductive age for family planning services, and people living with HIV infections who seek care at study sites.

- Includes facility-based consultation fees, medications, supplies, and services provided at $\mathrm{IH}$ intervention sites and advanced care referrals at the district or regional hospital.

- Selected fees and coverage population chosen in consultation with $\mathrm{MoH}$ and based on national and global guidelines.

- Candidate selection from community by local leadership, health facility, and $\mathrm{IH}$ staff.

- Preference for female residents who meet selection criteria (some literacy, pre-test/post-test results, demonstrated related competencies).

- Pre-service 23-day training in IMCl, maternal health, and HIV counseling and testing led by $\mathrm{MoH}$ and $\mathrm{IH}$ staff.

- In-service 5-day training in family planning and counseling led by $\mathrm{MoH}$ and $\mathrm{IH}$ staff.

- All training materials developed in consultation with $\mathrm{MoH}$ and based on national/global guidelines and evidence-based materials from Association Togolaise pour le Bien-Être Familial, Better Birth Project, Last Mile Health, Muso, and Partners In Health.

- Equipped with materials (training guides, backpacks, timers, thermometers, scales, MUACs, rapid tests, medical treatment for basic $\mathrm{IMCl}$ cases, notebooks, pens).

- CWH consultations, referrals, medicines, and materials are provided free of charge.

- Supportive supervision with coaching and mentoring by $\mathrm{IH}$ supervisor (nurse/medical assistant).

- Regular observation of CHW service delivery through routine programmatic data and community feedback.

- CHWs receive a regular equitable salary for full-time work through proactive case seeking and follow-up.
4. Clinical mentoring and enhanced supervision by a trained peer coach at public sector health facilities Evidence: [28-30]
5. Basic infrastructure/equipment improvements and supply chain management training of pharmacy managers Evidence: [15, 31-33]
- Onsite pre-service 4-day training in maternal, reproductive, neonatal, and child health and HIV led by IH clinical mentor (nurse/medical assistant) and medical director.

- Training materials developed in collaboration with $\mathrm{MoH}$ and based on national/global guidelines and evidence-based materials from WHO, American Academy of Pediatrics, Ariadne Labs, Better Birth Project, Last Mile Health, Muso, and Partners In Health.

- Weekly facility-based supportive supervision by IH clinical mentors (nurses, midwives, medical assistants) with prior experience in public sector health facilities.

- Regular observation of facility staff service delivery through routine programmatic data and community feedback.

- Formal infrastructure assessment and equipment needs with $\mathrm{MoH}$ using WHO's SARA tool [41].

- Facilitate structural improvements to improve care delivery.

- Equip facility with essential medicines and equipment identified by assessment and national health protocols.

- Onsite training in supply-chain management practices, including proper storage of medicines, filling of stock cards, and orders based on average monthly input consumption.

- Regular supervision and support by $I \mathrm{H}$ clinical mentor. generalizable data that could inform replication and scale activities.

To address these limitations and rigorously assess ICBHSS effectiveness and implementation strategy, we will conduct a summative mixed-methods evaluation using a modified REAIM (reach, effectiveness, adoption, implementation, maintenance) framework [42]. This framework is a practical tool that assesses complex interventions in real-world practice 
including measures of clinical effectiveness and implementation strategy [43]. Effectiveness will be measured using the primary outcome, under-five child mortality, utilizing a stepped-wedge cluster randomized control design. Implementation strategy will be assessed by measures of reach, adoption, implementation, and maintenance of the ICBHSS intervention through data collection with key informants, facility, and the general catchment populations [44]. This adapted evaluation framework will enhance traditional stepped-wedge design by including measures of implementation fidelity [45].

Given the baseline population need, preliminary effectiveness results, and $\mathrm{MoH}$ request for scale-up, we deem it unethical to withhold the intervention from any of the comparison sites. Logistical and financial constraints to launch the ICBHSS model already require the sequential implementation needed for a stepped-wedge trial. This protocol is the best fit considering the limitations from the initial pilot using a single-arm, repeated cross-sectional design and the need for generalizable findings to support translating results into practice $[45,46]$. Annual data collection, while an increased demand of resources and cost, was deemed both feasible and beneficial, as it will allow regular quantitative and qualitative data analysis and iterative dissemination of results, internally to support programmatic improvements, and with $\mathrm{MoH}$ partners for embedded research practices [47]. Our objective in this paper is to describe the research protocol of this pragmatic hybrid effectiveness-implementation study to measure the effectiveness and implementation strategy of the ICBHSS initiative over 48 months in rural northern Togo using an adapted RE-AIM framework.

\section{Methods/design}

\section{Study aims}

The objective of this study is to optimize ICBHSS model implementation using the adapted RE-AIM evaluation framework. To achieve this, we have two primary objectives: (1) to determine the effectiveness of the ICBHSS model and (2) to assess the implementation strategy through measurements of reach, adoption, implementation, and maintenance. Our specific study aims include the following:

Primary aim 1: effectiveness

(1) Analyze longitudinal changes in maternal and child mortality and morbidity, quality of care parameters, and public sector facility readiness in catchment areas.

Primary aim 2: implementation strategy

(1) Identify barriers and facilitators contributing to access and quality of ICBHSS services;

(2) Measure changes in coverage, health service utilization rates, and intervention adoption;
(3) Determine ICBHSS implementation costs and return-on-investment estimates.

\section{Design}

This study uses a pragmatic type II hybrid effectivenessimplementation design [35] to evaluate the two primary aims of effectiveness and implementation strategy by the ICBHSS initiative using a modified RE-AIM implementation science framework [42]. See Additional file 1 for the CONSORT checklist. We will include four distinct study components: (1) a stepped-wedge cluster randomized control trial using a community-based household survey, (2) annual health facility assessments at each selected site, (3) qualitative key informant interviews conducted 1-year post-intervention reception, and (4) annual costing and return-on-investment analyses using the Community Health and Costing Tool [48] and the Lives Saved Tool (LiST) [49]. Further details about each study component are described below with Table 2 summarizing a timeline.

\section{Stepped-wedge cluster randomized control trial}

This pragmatic design leverages the sequential or staggered rollout of the ICBHSS model and will facilitate an assessment of effectiveness as well as implementation strategy through coverage and adoption metrics by comparing geographically organized clusters [50, 51]. It includes four clusters that organize intervention health facilities by district: Bassar, Binah, Dankpen, and Kéran. The ICBHSS cluster initiation order will be randomized annually with four steps, as it was independently determined by the IH programs team that baseline health needs were similar in each cluster. Community-based household surveys adapted from Demographic and Health Survey (DHS) modules previously implemented in Togo [3] and focusing on demographic, maternal, and child health data will be conducted in each cluster at baseline, 12, 24, 36, and 48 months.

\section{Health facility assessments}

These assessments will employ facility-level surveys based on the World Health Organization (WHO) Service Availability and Readiness Assessment tool (SARA) [41] and will provide effectiveness information about facility-level service quality. Surveys will be completed for each health facility annually at baseline, 12, 24, 36, and 48 months.

\section{Key informant interviews}

Qualitative interviews will be completed with key informants to assess barriers and facilitators to program implementation fidelity and feasibility while also documenting contextual factors. The first key informant interviews will be conducted 12 
Table 2 Data collection and Integrated Community-Based Health Systems Strengthening (ICBHSS) initiation timeline based on staggered implementation*

\begin{tabular}{|c|c|c|c|c|c|c|c|c|c|c|}
\hline Cluster & Activity** & $\begin{array}{c}\text { Months } \\
\mathbf{1 - 2} \\
\text { May/June } \\
2018\end{array}$ & $\begin{array}{c}\text { Months } \\
\mathbf{3 - 1 2} \\
\text { July } \\
2018- \\
\text { April } \\
2019\end{array}$ & $\begin{array}{c}\text { Months } \\
\text { 13-14 } \\
\text { May/June } \\
2019\end{array}$ & $\begin{array}{c}\text { Months } \\
15-24 \\
\text { July } 2019 \\
\text { - April } \\
2020\end{array}$ & $\begin{array}{c}\text { Months } \\
\mathbf{2 5 - 2 6} \\
\text { May/June } \\
2020\end{array}$ & $\begin{array}{c}\text { Months } \\
\text { 27-36 } \\
\text { July } \\
2020- \\
\text { April } \\
2021\end{array}$ & $\begin{array}{c}\text { Months } \\
\text { 37-38 } \\
\text { May/June } \\
2021\end{array}$ & $\begin{array}{c}\text { Months } \\
\text { 39-48 } \\
\text { July } \\
2021- \\
\text { April } \\
2022\end{array}$ & $\begin{array}{c}\text { Months } \\
\text { 49-50 } \\
\text { May/June } \\
2022\end{array}$ \\
\hline \multirow[t]{5}{*}{ District 1} & $\begin{array}{l}\text { ICBHSS } \\
\text { start }\end{array}$ & $\mathbf{X}$ & $\mathbf{X}$ & $\mathbf{X}$ & $\mathbf{X}$ & $\mathbf{X}$ & $\mathbf{X}$ & $\mathbf{X}$ & $\mathbf{X}$ & $\mathbf{X}$ \\
\hline & $\mathrm{CH}$ survey & $\mathbf{X}$ & & $\mathbf{X}$ & & $\mathbf{X}$ & & $\mathbf{X}$ & & $\mathbf{X}$ \\
\hline & Fac survey & $\mathbf{X}$ & & $\mathbf{X}$ & & $\mathbf{X}$ & & $X$ & & $\mathbf{X}$ \\
\hline & Kls & & $X$ & & $\mathbf{X}$ & & $\mathbf{X}$ & & $X$ & \\
\hline & Cost survey & & $X$ & & $\mathbf{X}$ & & $X$ & & $X$ & \\
\hline \multirow[t]{5}{*}{ District 2} & $\begin{array}{l}\text { ICBHSS } \\
\text { start }\end{array}$ & & & $\mathbf{X}$ & $\mathbf{X}$ & $\mathbf{X}$ & $\mathbf{X}$ & $X$ & $X$ & $X$ \\
\hline & $\mathrm{CH}$ survey & $\mathbf{X}$ & & $\mathbf{X}$ & & $\mathbf{X}$ & & $\mathbf{X}$ & & $\mathbf{X}$ \\
\hline & Fac survey & $\mathbf{X}$ & & $\mathbf{X}$ & & $\mathbf{X}$ & & $\mathbf{X}$ & & $\mathbf{X}$ \\
\hline & $\mathrm{Kls}$ & & & & $\mathbf{X}$ & & $\mathbf{X}$ & & $\mathbf{X}$ & \\
\hline & Cost survey & & & & $\mathbf{X}$ & & $\mathbf{X}$ & & $\mathbf{X}$ & \\
\hline \multirow[t]{5}{*}{ District 3} & $\begin{array}{l}\text { ICBHSS } \\
\text { start }\end{array}$ & & & & & $\mathbf{X}$ & $\mathbf{X}$ & $\mathbf{X}$ & $\mathbf{X}$ & $\mathbf{X}$ \\
\hline & $\mathrm{CH}$ survey & $\mathbf{X}$ & & $\mathbf{X}$ & & $\mathbf{X}$ & & $\mathbf{X}$ & & $\mathbf{X}$ \\
\hline & Fac survey & $X$ & & $\mathbf{X}$ & & $X$ & & $X$ & & $\mathbf{X}$ \\
\hline & $\mathrm{KIs}$ & & & & & & $\mathbf{X}$ & & $\mathbf{X}$ & \\
\hline & Cost survey & & & & & & $\mathbf{X}$ & & $\mathbf{X}$ & \\
\hline \multirow[t]{5}{*}{ District 4} & $\begin{array}{l}\text { ICBHSS } \\
\text { start }\end{array}$ & & & & & & & $\mathbf{X}$ & $\mathbf{X}$ & $\mathbf{X}$ \\
\hline & $\mathrm{CH}$ survey & $\mathbf{X}$ & & $\mathbf{X}$ & & $\mathbf{X}$ & & $\mathbf{X}$ & & $\mathbf{X}$ \\
\hline & Fac survey & $\mathbf{X}$ & & $\mathbf{X}$ & & $\mathbf{X}$ & & $\mathbf{X}$ & & $\mathbf{X}$ \\
\hline & KIs & & & & & & & & $\mathbf{X}$ & $\mathbf{X}$ \\
\hline & Cost survey & & & & & & & & $\mathbf{X}$ & \\
\hline
\end{tabular}

*Follows the CONSORT extension diagram for stepped-wedge cluster randomized trials [52]

${ }^{* *} \mathrm{CH}$ community-based household survey, Fac health facility assessments, Kls key informant interviews, Cost survey costing and return-on-investment assessment

months post-intervention start and at subsequent 12-month intervals until study end within each cluster.

\section{Costing and return-on-investment assessment}

ICBHSS program costs and return on investment will be measured using the Community Health Planning and Costing Tool [48] and the Lives Saved Tool [49] to assess implementation strategy approaches and to inform considerations of maintenance and national planning efforts. The first assessment will be conducted 12 months post-intervention start and at annual subsequent 12-month intervals until study end within each cluster.

\section{Study setting}

The study will be conducted in the catchment areas of 21 public sector health facilities within the Kara region's rural districts of Bassar, Binah, Dankpen, and Kéran. The total population is approximately 181,111 people. Study sites were selected by $\mathrm{MoH}$ partners and $\mathrm{IH}$ programmatic staff based on perceived population health needs, ongoing regional public-private program activities, and population size. All selected sites are primary healthcare facilities operated by the $\mathrm{MoH}$ [53] that serve rural populations. Estimated catchment population and utilization rates for these sites are listed by district in Table 3. As described in Table 2, the ICBHSS initiative will be sequentially implemented by district each year within these 21 preselected sites.

\section{Inclusion criteria}

Eligibility for inclusion is described below by study component for the community-based household surveys, facility surveys, and qualitative interviews.

\section{Community-based household surveys}

Females 15-49years of age who reside in a selected household within the study catchment area are included. Participants $15-17$ years of age will only be included if they have been/are currently pregnant and have a waiver of parental permission. Households will be randomly selected each year using a systematic population-weighted sampling methodology. If there is more than one eligible respondent in the household, one will be randomly selected based on a Kish selection grid [54]. All participants will have the opportunity to decline participation during the informed consent process. 
Table 3 List of study sites $(N=21)$ with estimated baseline catchment population $(N=181,111)$ and facility utilization rates

\begin{tabular}{|c|c|c|c|}
\hline District & Study site & Catchment population* & Facility utilization rate** \\
\hline Bassar & Bangéli & 16,169 & $42 \%$ \\
\hline Bassar & Kabou-Sara & 10,054 & $56 \%$ \\
\hline Bassar & Koundoum & 7,428 & $37 \%$ \\
\hline Bassar & Manga & 5,006 & $28 \%$ \\
\hline Bassar & Sanda-Afohou & 5,514 & $43 \%$ \\
\hline Binah & Asseré & 4,446 & $19 \%$ \\
\hline Binah & Boufalé & 4,212 & $48 \%$ \\
\hline Binah & Kouyorira & 4,364 & $26 \%$ \\
\hline Binah & N'Djei & 3,258 & $53 \%$ \\
\hline Binah & Pessaré & 8,002 & $26 \%$ \\
\hline Binah & Sirka & 5,980 & $51 \%$ \\
\hline Binah & Solla & 5,960 & $58 \%$ \\
\hline Dankpen & Koutière & 13,097 & $12 \%$ \\
\hline Dankpen & Kpétab & 8,208 & $22 \%$ \\
\hline Dankpen & Naware & 19,531 & $10 \%$ \\
\hline Dankpen & Solidarité & 8,157 & $24 \%$ \\
\hline Kéran & Kokou-Temberma & 8,722 & Unknown \\
\hline Kéran & Nadoba & 16,593 & Unknown \\
\hline Kéran & Natiponi & 7,636 & Unknown \\
\hline Kéran & Pangouda & 10,735 & Unknown \\
\hline Kéran & Warengo & 8,039 & Unknown \\
\hline
\end{tabular}

*Data derived from 2016 Ministry of Health population estimates and 2018 Integrate Health baseline population-based sampling **Data source is 2016 Ministry of Health district-level annual reports

\section{Health facility surveys}

All 21 selected health facilities will be surveyed. See Table 3 for facility details.

\section{Key informant interviews}

This component will include individuals 18 years of age or older who are either implementing partners or intervention health facility staff. Implementing partners are defined as $\mathrm{IH}$ programmatic staff members employed in the catchment area of interest. Health facility staff are defined as clinical or administrative $\mathrm{MoH}$ employees working at one of the 21 health centers. Approximately 42 key informants from the 21 health facilities (21 implementing partners and 21 clinical health facility staff) will be included.

\section{Sample size determination}

This study is powered to detect a change by cluster in under-five mortality per 1000 live births. A sample size of 7600 participants will provide $80 \%$ power to detect an estimated $30 \%$ reduction or greater of under-five mortality from the estimated baseline of 70 per 1000 live births, with an alpha of 0.05 , intracluster correlation of 0.005 , $20 \%$ non-response rate, and estimated 0.5 children under five per participant $[9,55]$. Effect size is a conservative estimate based on past pilot experience (ClinicalTrials. gov Identifier: NCT03773913).

\section{Randomization}

The cluster order for implementation at each step will be determined randomly by an external technical advisor using a random number generator. Randomization will occur each year 8 months prior to the rollout of the intervention in the next cluster. This will enable blinding to the random order of clusters for $\mathrm{IH}$ and $\mathrm{MoH}$ staff 7involved in implementation while also allowing for an annual 8-month planning stage prior to start of the intervention. Each cluster represents a district, with a total of 21 preselected health facilities across each of the four districts.

\section{Data collection and analysis}

All metrics will be organized using a modified RE-AIM (reach, effectiveness, adoption, implementation, maintenance) framework [56]. Table 4 summarizes data collection and analysis plans organized by primary aim and adapted RE-AIM domains. 
Table 4 Summary of key study measures organized by aim and domain using modified RE-AlM evaluation framework

\begin{tabular}{lll}
\hline Study aim & $\begin{array}{l}\text { RE-AIM } \\
\text { framework } \\
\text { domain }\end{array}$ & Outcome(s)/indicator(s) \\
\hline $\begin{array}{l}\text { Study aim I: } \\
\text { implementation } \\
\text { strategy }\end{array}$ & Reach & Health service coverage \\
& & CHW home visits
\end{tabular}

Study aim 2: effectiveness

\section{Effectiveness}

\section{Primary outcome}

Children under five mortality rate
CHW home treatment

Health facility treatment

Early service access for child health

Malaria coverage

Pneumonia coverage

Gastrointestinal illness coverage

Malnutrition coverage

Coverage estimate of prenatal care

Percentage of births at healthcare facility

Percentage of births at home

Coverage estimate of post-natal care

Community-

based

household

survey

Community-

based

household

survey

Community-

based

household

survey

Community-

based

household

survey

Community-

based

household

survey

Community-

based

household

survey

Community-

based

household

survey

Community-

based

household

survey

Community-

based

household

survey

Community-

based

household

survey

Community-

based

household

survey

Community-

based

household

survey

Secondary outcomes

Neonatal mortality rate
Proportion of participants in the last year reporting a home visit by an $\mathrm{H} \mathrm{CHW}$.

Proportion of participants in the last year reporting treatment at home by an $\mathrm{H} \mathrm{CHW}$

Proportion of participants in the last year reporting care at a health facility.

Proportion of children under five reported febrile receiving guideline-based treatment within $24 \mathrm{~h}$ of symptom onset.

Proportion of children under five with a cough and proportion of those receiving guideline-based treatment within $24 \mathrm{~h}$ of symptom onset.

Proportion of children under five with diarrhea receiving guideline-based treatment within $24 \mathrm{~h}$ of symptom onset.

Proportion of children under five with malnutrition receiving effective treatment.

Proportion of pregnant women in the last two years who completed four ANC visits.

Proportion of pregnant women in the last two years who delivered in a health facility

Proportion of pregnant women who delivered at home in last 2 years.

Proportion of post-partum women who received postnatal care in last 2 years.

Using a standard birth/death history table, calculate under-five mortality rates, and compare risk of death before age five across surveys with the Cox proportional hazards regression using survey year as the explanatory variable. Children still alive and under age five at the time of survey will be right censored.

Community- The neonatal mortality rates from all births reported by based respondents in the 5 years prior to the survey using the household same methods described for under-five mortality, 
Table 4 Summary of key study measures organized by aim and domain using modified RE-AIM evaluation framework (Continued)

\begin{tabular}{|c|c|c|c|c|}
\hline Study aim & $\begin{array}{l}\text { RE-AIM } \\
\text { framework } \\
\text { domain }\end{array}$ & Outcome(s)/indicator(s) & Data source & Indicator definition/clarification \\
\hline & & & survey & adjusted for 28 days. \\
\hline & & Children under one mortality rate & $\begin{array}{l}\text { Community- } \\
\text { based } \\
\text { household } \\
\text { survey }\end{array}$ & $\begin{array}{l}\text { The under-one mortality rates from all births reported by } \\
\text { respondents in the } 5 \text { years prior to the survey using the } \\
\text { same methods described for under-five, adjusted for } 1 \\
\text { year. }\end{array}$ \\
\hline & & Children under two mortality rate & $\begin{array}{l}\text { Community- } \\
\text { based } \\
\text { household } \\
\text { survey }\end{array}$ & $\begin{array}{l}\text { The under-two mortality rates from all births reported by } \\
\text { respondents in the } 5 \text { years prior to the survey using the } \\
\text { same methods described for under-five, adjusted for } 2 \\
\text { years. }\end{array}$ \\
\hline & & Maternal mortality rate & $\begin{array}{l}\text { Community- } \\
\text { based } \\
\text { household } \\
\text { survey }\end{array}$ & $\begin{array}{l}\text { Exploratory maternal mortality analysis based on the } \\
\text { sisterhood reports }[57,58] \text {. }\end{array}$ \\
\hline & & Quality of care parameters & & \\
\hline & & $\begin{array}{l}\text { Timeliness/promptitude of child care } \\
\text { for malaria }\end{array}$ & $\begin{array}{l}\text { Routine } \\
\text { programmatic } \\
\text { data }\end{array}$ & $\begin{array}{l}\text { Proportion of children under five reported febrile and } \\
\text { the proportion who received effective antimalarial } \\
\text { treatment within } 24 \mathrm{~h} \text { of symptom onset. }\end{array}$ \\
\hline & & $\begin{array}{l}\text { Timeliness/promptitude of child care } \\
\text { for pneumonia }\end{array}$ & $\begin{array}{l}\text { Routine } \\
\text { programmatic } \\
\text { data }\end{array}$ & $\begin{array}{l}\text { Proportion of children under five reported with cough } \\
\text { and the proportion of those children who received an } \\
\text { effective pneumonia treatment within } 24 \mathrm{~h} \text { of symptom } \\
\text { onset. }\end{array}$ \\
\hline & & $\begin{array}{l}\text { Timeliness/promptitude of child care } \\
\text { for diarrhea }\end{array}$ & $\begin{array}{l}\text { Routine } \\
\text { programmatic } \\
\text { data }\end{array}$ & $\begin{array}{l}\text { Proportion of children under five reported with diarrhea } \\
\text { and the proportion of those children who received an } \\
\text { effective treatment for diarrheal disease within } 24 \mathrm{~h} \text { of } \\
\text { symptom onset. }\end{array}$ \\
\hline & & CHW technical competence & $\begin{array}{l}\text { Routine } \\
\text { programmatic } \\
\text { data }\end{array}$ & $\begin{array}{l}\text { Proportion of } \mathrm{IH} \text { CHWs who adhere to evidence-based } \\
\text { protocols for iCCM and maternal health. }\end{array}$ \\
\hline & & $\begin{array}{l}\text { Facility clinical staff technical } \\
\text { competence }\end{array}$ & $\begin{array}{l}\text { Routine } \\
\text { programmatic } \\
\text { data }\end{array}$ & $\begin{array}{l}\text { Proportion of facility clinical staff who adhere to } \\
\text { evidence-based protocols for iCCM and maternal health. }\end{array}$ \\
\hline & & Equitable & $\begin{array}{l}\text { Community- } \\
\text { based } \\
\text { household } \\
\text { survey }\end{array}$ & $\begin{array}{l}\text { Access differences in child mortality between maternal } \\
\text { wealth quintiles, distance to facility, and education level. }\end{array}$ \\
\hline & & Healthcare readiness score & $\begin{array}{l}\text { Health facility } \\
\text { assessments }\end{array}$ & $\begin{array}{l}\text { Examine facility changes in procurement, physical } \\
\text { infrastructure, and management through annual } \\
\text { readiness score [41]. }\end{array}$ \\
\hline
\end{tabular}

Study aim I: $\quad$ Adoption implementation strategy
Community-level engagement

Community engagement sessions

Participant-level behavior change

Child care cascades for fever, pneumonia, and diarrhea

Women of reproductive age cascade for antenatal care, facility-based delivery, and post-natal care
Community-

based household survey
Routine Number of community forums and community members programmatic in attendance.

data

Community- Changes in childcare-seeking behavior over time for based fever, pneumonia, and diarrhea in patients presenting to household health clinic, CHW, or non-clinical site.

survey Test if these proportions increased using the same approach to mixed-effects generalized linear models as described in the primary effectiveness outcome measure.

Changes in pregnant women care-seeking behavior over time, services delivered by IH CHWs, facility-based delivery, as well as antenatal care and post-natal care attendance. Test if these proportions increased using the same approach to mixed effects generalized linear models as described in the primary effectiveness outcome measure. 
Table 4 Summary of key study measures organized by aim and domain using modified RE-AIM evaluation framework (Continued)

\begin{tabular}{|c|c|c|c|c|}
\hline Study aim & $\begin{array}{l}\text { RE-AIM } \\
\text { framework } \\
\text { domain }\end{array}$ & Outcome(s)/indicator(s) & Data source & Indicator definition/clarification \\
\hline \multirow{5}{*}{$\begin{array}{l}\text { Study aim I: } \\
\text { implementation } \\
\text { strategy }\end{array}$} & \multirow[t]{5}{*}{ Implementation } & \multicolumn{3}{|l|}{ Qualitative interviews } \\
\hline & & Fidelity & $\begin{array}{l}\text { Key informant } \\
\text { interviews }\end{array}$ & $\begin{array}{l}\text { Degree that intervention(s) were implemented as } \\
\text { planned in original protocol. }\end{array}$ \\
\hline & & Feasibility & $\begin{array}{l}\text { Key informant } \\
\text { interviews }\end{array}$ & $\begin{array}{l}\text { Extent that an intervention can be carried out in a } \\
\text { particular setting. }\end{array}$ \\
\hline & & Outer context [59] & $\begin{array}{l}\text { Key informant } \\
\text { interviews }\end{array}$ & $\begin{array}{l}\text { Macro-level external factors including social, funding, and } \\
\text { leadership. }\end{array}$ \\
\hline & & Inner context [59] & $\begin{array}{l}\text { Key informant } \\
\text { interviews }\end{array}$ & $\begin{array}{l}\text { Micro-level internal factors including } \mathrm{IH} / \mathrm{MoH} \text { partnership, } \\
\text { distinct issues about IH and MoH roles, feedback, facility, } \\
\text { community, household, and individual level. }\end{array}$ \\
\hline \multirow{3}{*}{$\begin{array}{l}\text { Study aim I: } \\
\text { implementation } \\
\text { strategy }\end{array}$} & \multirow[t]{3}{*}{ Maintenance } & \multicolumn{3}{|c|}{ Costing and return-on-investment assessment } \\
\hline & & Annual price per capita & $\begin{array}{l}\text { Costing } \\
\text { surveys }\end{array}$ & $\begin{array}{l}\text { Price per capita compared to current } \mathrm{MoH} \text { funding using } \\
\text { the Community Health Planning and Costing Tool [48] at } \\
\text { the cluster level. }\end{array}$ \\
\hline & & Return on investment & $\begin{array}{l}\text { Costing } \\
\text { surveys }\end{array}$ & $\begin{array}{l}\text { Return on investment using the Community Health } \\
\text { Planning and Costing Tool [48] and the Lives Saved Tool } \\
\text { [49] with primary outcome at the cluster level. }\end{array}$ \\
\hline
\end{tabular}

\section{Primary aim 1: effectiveness}

Effectiveness We define effectiveness metrics as those that assess the impact of the ICBHSS initiative using annual community household surveys, routine programmatic data, and the health facility assessments. The community-level primary outcome by district uses underfive mortality rates as well as the secondary outcomes of neonatal, under-one, under-two, and maternal mortality rates. We will additionally evaluate quality of care parameters focusing on timeliness of child healthcare through promptitude of treatment reception following illness diagnosis and health facility readiness scores. Lastly, we will assess equity through secondary analyses of under-five mortality by household wealth quintiles, maternal education levels, and distance from the nearest health facility.

Primary outcome: under-five mortality rates. We will calculate under-five mortality rates from all births reported by respondents using a standard birth/death history table. We will calculate under-five mortality rates and compare the risk of death before age five across surveys with the Cox proportional hazards regression using intervention exposure as the explanatory variable. Children still alive and under age five at the time of survey will be right censored.

Secondary outcomes: neonatal, under-one, and undertwo mortality rates. We will calculate the neonatal, under-one, and under-two mortality rates from all births reported by respondents using the same methods described above adjusted for 28 days, 1 year, and 2 years.

Secondary outcome: maternal mortality. We will calculate an exploratory maternal mortality analysis based on sisterhood reports [57, 58].

\section{Primary aim 2: implementation strategy}

Reach We define reach metrics as the proportion of target population that gained access to the ICBHSS initiative services using the annual community household surveys. We will assess the implementation strategy through individual-level ICBHSS participation with communitylevel (vis-à-vis CHWs) and facility-level health service utilization using health service coverage estimates and early service access for child health. We will use a mixedeffects generalized linear model to compare preintervention to post-intervention proportions for each reach metric while adjusting for clustering at the facility and district level and time and allowing for district-level estimates to be random effects. Our primary analysis will not include adjustment for individual-level characteristics, as each district will serve as its own control.

Adoption We define adoption metrics for this study as the proportion of the community and providers changing health-seeking or providing behavior. Our evaluation of adoption will be completed using routine programmatic data and annual community household surveys. Through this domain, we will assess implementation strategy measures using community-level engagement and behavior change by the individual-level uptake of the ICBHSS intervention. We will test whether these proportions increased, applying the same approach described in the reach evaluation measures through mixed-effects generalized linear models.

Implementation Metrics of implementation are expressed as fidelity and feasibility as well as 
documenting contextual factors [59]. Evaluation of implementation will be completed using key informant qualitative assessments (in-depth interviews with implementing partners and health facility staff) that will be conducted 1-year post-intervention at the cluster (district) level. It will complement quantitative data collected to evaluate implementation strategy and will assess emerging themes.

Maintenance Our study defines maintenance metrics as costs required to deliver and sustain the ICBHSS model. Evaluation of maintenance will be completed using the costing and return-on-investment analysis, which will be conducted each year of implementation at the cluster (district) level. This analysis will assess program implementation costs based on strategy design retrospectively using the Community Health Planning and Costing Tool [48] and the Lives Saved Tool [49]. These results will be used to inform planning and policy decisions and processes. Lastly, to further triangulate the validity of our findings, we will compare our baseline and 36-month estimates for the domains of reach, effectiveness, and adoption to the most recent Togo DHS or Multiple Indicator Cluster Survey (MICS) data. We will furthermore compare the domains of reach and adoption to ICBHSS through programmatic data collected at the community and facility level.

\section{Dissemination plan}

We will routinely disseminate study data with key stakeholders in Togo at the national, subnational, and community level, as well as the global community of public health practitioners, researchers, and policymakers. IH staff will conduct biannual forums with local leaders, public sector health facility staff, and community members to discuss ICBHSS implementation and share findings. Based on these forums, $\mathrm{IH}$ and $\mathrm{MoH}$ partners will collaboratively decide to adapt the implementation strategy and, if deemed necessary, the intervention. IH staff will additionally participate in $\mathrm{MoH}$ monthly districtlevel meetings for district health personnel to stay current in $\mathrm{MoH}$ plans and to share findings from ICBHSS initiatives. Results will be published in conference abstracts and peer-reviewed journals with preference for publicly available publications in collaboration with partners at the Togolese $\mathrm{MoH}$.

\section{Discussion}

We have described our rationale, study design, and implementation strategy details regarding this pragmatic type II hybrid design to serve as a model for those interested in pragmatic implementation studies that allow for continuous intervention improvement.
This study design includes several limitations, including limitations with any real-world pragmatic steppedwedge trial. There are concerns related to confounding, bias, and temporal trends that may limit the validity of our findings. We used a modest effect size, a cluster randomization, and an analysis plan to mitigate these limitations. There are contextual factors that may be challenging to delineate that influence our primary outcomes. We attempted to minimize this at baseline through site selection.

In spite of these limitations, this study will enable a rigorous evaluation in a real-world setting that measures effectiveness and implementation strategy, while also contributing to knowledge generation to inform and complement national health strategies. A one-size-fits-all approach for evaluation does not work for the implementation of multiple interventions and corresponding strategies, particularly in real-world settings. We intend to provide an effective intervention accompanied by an implementation roadmap that includes enabling and non-enabling factors. Through this directed scientific inquiry and mixed-methods evaluation, we aim to contribute to knowledge and foster partnerships that improve quality and access to community health systems in Togo and beyond.

\section{Additional file}

Additional file 1: CONSORT checklist of information to include when reporting a stepped-wedge cluster randomized trial (SW-CRT). (DOCX $18 \mathrm{~kb}$ )

Additional file 2: French translation of manuscript text with included figures and tables. (DOCX $218 \mathrm{~kb}$ )

\section{Abbreviations}

CBO: Community-based organization; CHSL: Community Health Systems Lab; CHW: Community health worker; DHS: Demographic and Health Survey; EPI: Expanded Program on Immunization; ICBHSS: Integrated CommunityBased Health Systems Strengthening model; iCCM: Integrated Community Case Management; IH: Integrate Health; IMCl: Integrated Management of Childhood IIIness; LiST: Lives Saved Tool; MICS: Multiple Indicator Cluster Surveys; MoH: Ministry of Health and Public Hygiene; NGO: Nongovernmental organization; RE-AIM: Reach, Effectiveness, Adoption, Implementation, Maintenance Framework; SARA: Service Availability and Readiness Assessment; SDG: Sustainable Development Goal; SWCRT: Stepped-wedge cluster randomized trial; WHO: World Health Organization

\section{Acknowledgements}

We acknowledge and thank the following partners and advisors for their integral assistance in developing and implementing this study : the health authorities in the Kara region and the districts of Bassar, Binah, Dankpen, and Kéran; $I H$ staff in Togo and New York; the Albert Einstein College of Medicine; and the University of Washington.

Translated article

The French version of this article is provided in Additional file 2.

Authors' contributions

All authors developed the study design. MEL, KPF, HEJ, SCG, KDL, and LRH drafted and revised the study protocol. MEL, KPF, and HEJ developed the 
analytic and statistical plan. All authors participated in the study implementation and have read and approved the final manuscript.

\section{Funding}

Study funded by $\Vdash$. No funders had any role in the design of this study.

\section{Availability of data and materials}

The associated study protocol and data collection tools will be made available upon request from the corresponding author. Quantitative datasets are available from the corresponding author upon reasonable request after the completion of primary analyses and results dissemination. Qualitative study datasets will not be available, as they may include identifiable information that could comprise participant identity.

\section{Ethics approval and consent to participate}

Ethics approvals for this study were obtained from the institutional review boards of the Togolese Ministry of Health in Lomé, Togo (ref: CRBS/33/2017) and the Albert Einstein College of Medicine in New York, USA (ref: 039328). Informed consent will be obtained from all study participants over 18 years of age, and a waiver of parental permission will be obtained for 15- to 17year-old participants. In cases of refusal, the individuals will not be included in the study.

\section{Consent for publication}

Not applicable.

\section{Competing interests}

The authors declare that they have no competing interests.

\section{Author details}

${ }^{1}$ Community Health Systems Lab, Integrate Health/Santé Intégrée, Kara, Togo. ${ }^{2}$ Department of Pediatrics, Albert Einstein College of Medicine, Bronx, NY, USA. ${ }^{3}$ Department of Family and Social Medicine, Albert Einstein College of Medicine, Bronx, NY, USA. ${ }^{4}$ CUNY Graduate School of Public Health \& Health Policy, New York, USA. ${ }^{5}$ Integrate Health/Santé Intégrée, Kara, Togo. ${ }^{6}$ Kara Regional Health Department, Ministry of Health and Public Hygiene, Kara, Togo. ${ }^{7}$ Department of Pediatrics, Health Sciences Faculty, University of Lomé, Lomé, Togo. ${ }^{8}$ Department of Pediatrics, Regional Hospital, Lomé-Commune, Lomé, Togo. ${ }^{9}$ Northwestern University Feinberg School of Medicine, Chicago, USA.

\section{Received: 23 May 2019 Accepted: 1 July 2019}

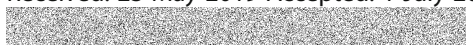

\section{References}

1. World Health Organization. World Health Statistics 2015. 2015

2. Golding N, Burstein R, Longbottom J, Browne AJ, Fullman N, OsgoodZimmerman A, et al. Mapping under-5 and neonatal mortality in Africa, 2000-15: a baseline analysis for the Sustainable Development Goals. Lancet (London, England) [Internet]. 2017 [cited 2018 Oct 22];390(10108):21712182. Available from: http://www.ncbi.nlm.nih.gov/pubmed/28958464.

3. Ministère de la Planification, du Développement et de l'Aménagement du Territoire (MPDAT), Ministère de la Santé (MS) et ICF International. Enquête Démographique et de Santé au Togo 2013-2014. Rockville: MPDAT, MS et ICF International; 2015

4. UN Inter-agency Group for Child Mortality Estimation (UN IGME). Countryspecific under-five mortality rate, 2018 [Internet]. 2018 [cited 2018 Dec 1] Available from: https:/data.unicef.org/topic/child-survival/under-five-mortality/.

5. Togo Ministry of Health. Togo Ministère de la Santé: Rapport Annuel de Performance Gestion 2014. 2014.

6. Togo Ministry of Health. Togo Ministère de la Santé: Principaux Indicateurs de Sante 2014. 2014

7. Fiori K, Schechter J, Dey M, Braganza S, Rhatigan J, Houndenou S, et al. Closing the delivery gaps in pediatric HIV care in Togo, West Africa: using the care delivery value chain framework to direct quality improvement. AIDS Care. 2016;28(September 2017):29-33.

8. Fiori KP, Belli HM, Lauria ME, Hirschhorn LR, Schechter J, Hansman E, Rajshekhar N, Katin V, Gbeleou S, Grunitsky-Bekele M, Pitche VP. Implementing an integrated community based health systems strengthening approach to improve HIV survival in Northern Togo. AIDS Care. 2019;6:1.
9. McCarthy KJ, Braganza S, Fiori K, Gbeleou C, Kpakpo V, Lopez A, et al. Identifying inequities in maternal and child health through risk stratification to inform health systems strengthening in Northern Togo. PLoS One. 2017;12(3):1-19.

10. Arnold J, Samson M, Schechter J, Goodwin AS, Braganza S, Sesso GC, Lopez A, Fiori K. Getting there: overcoming barriers to reproductive and maternal health services access in Northern Togo-A Qualitative Study. World Medical \& Health Policy. 2016;8(3):223-44.

11. Johnson AD, Thomson DR, Atwood S, Alley I, Beckerman JL, Kone I, Diakite D, Diallo H, Traore B, Traore K, Farmer PE. Assessing early access to care and child survival during a health system strengthening intervention in Mali: a repeated cross sectional survey. PloS one. 2013;8(12):e81304.

12. Cometto G, Ford N, Pfaffman-Zambruni J, Akl EA, Lehmann U, McPake B, Ballard M, Kok M, Najafizada M, Olaniran A, Ajuebor O. Health policy and system support to optimise community health worker programmes: an abridged WHO guideline. The Lancet Global Health. 2018;6(12):e1397-404.

13. O'Mara-Eves A, Brunton G, McDaid D, Oliver S, Kavanagh J, Jamal F, et al. Community engagement to reduce inequalities in health: a systematic review, meta-analysis and economic analysis [Internet]. Community engagement to reduce inequalities in health: a systematic review, metaanalysis and economic analysis. NIHR J Libr; 2013 [cited 2019 Mar 18]. Available from: http://www.ncbi.nlm.nih.gov/pubmed/25642563.

14. O'Mara-Eves A, Brunton G, Oliver S, Kavanagh J, Jamal F, Thomas J. The effectiveness of community engagement in public health interventions for disadvantaged groups: a meta-analysis. BMC Public Health [Internet]. 2015 Dec 12 [cited 2019 Mar 18];15(1):129. Available from: http://www.ncbi.nlm. nih.gov/pubmed/25885588.

15. Kok MC, Dieleman M, Taegtmeyer M, Broerse JE, Kane SS, Ormel H, et al. Which intervention design factors influence performance of community health workers in low- and middle-income countries? A systematic review. Health Policy Plan [Internet]. 2015 Nov [cited 2019 Feb 9];30(9):1207-1227. Available from: http://www.ncbi.nlm.nih.gov/pubmed/25500559.

16. Yates R. Universal health care and the removal of user fees. Lancet (London, England) [Internet]. 2009 Jun 13 [cited 2019 Mar 15];373(9680):2078-2081. Available from: http://www.ncbi.nlm.nih.gov/pubmed/19362359.

17. Johri M, Ridde V, Heinmüller R, Haddad S. Estimation of maternal and child mortality one year after user-fee elimination: an impact evaluation and modelling study in Burkina Faso. Bull World Health Organ [Internet]. 2014 Oct 1 [cited 2018 Oct 31];92(10):706-715. Available from: https://www.ncbi. nlm.nih.gov/pmc/articles/PMC4208477/.

18. Calhoun LM, Speizer IS, Guilkey D, Bukusi E. The effect of the removal of user fees for delivery at public health facilities on institutional delivery in urban Kenya. Matern Child Health J [Internet]. 2017;0(0):0 Available from: https://www.ncbi.nlm.nih.gov/pmc/articles/PMC5845052/.

19. Johnson AD, Thiero O, Whidden C, Poudiougou B, Diakité D, Traoré F, et al. Proactive community case management and child survival in periurban Mali. BMJ Glob Heal Mali BMJ Glob Heal. 2018;33.

20. Schellenberg JRA, Adam T, Mshinda H, Masanja H, Kabadi G, Mukasa O, et al. Effectiveness and cost of facility-based Integrated Management of Childhood IIness (IMCI) in Tanzania. Lancet [Internet]. 2004 Oct 30 [cited 2019 Mar 18];364(9445):1583-1594. Available from: https://www. sciencedirect.com/science/article/pii/S014067360417311X.

21. Christopher J, Le MA. Review of the impact of community health workers delivering curative interventions against malaria, pneumonia and diarrhoea on child mortality and morbidity in. ... Resour Heal. 2011:1-11.

22. Lassi ZS, Bhutta ZA. Community-based intervention packages for reducing maternal and neonatal morbidity and mortality and improving neonatal outcomes. Cochrane Database Syst Rev [Internet]. 2015 Mar 23 [cited 2019 Mar 18];(3). Available from: https://doi.org/10.1002/14651858.CD007754. pub3.

23. Amouzou A, Morris S, Moulton LH, Mukanga D. Assessing the impact of integrated community case management (iCCM) programs on child mortality: review of early results and lessons learned in sub-Saharan Africa. J Glob Health [Internet]. 2014 Dec [cited 2019 Mar 18];4(2):020411. Available from: http://www.ncbi.nlm.nih.gov/pubmed/25520801.

24. Gogia S, Sachdev HS. Home visits by community health workers to prevent neonatal deaths in developing countries: a systematic review. Bull World Health Organ [Internet]. 2010 Sep 1 [cited 2019 Mar 18];88(9):658-666B. Available from: http://www.ncbi.nlm.nih.gov/pubmed/20865070.

25. Gogia S, Sachdev HPS. Home-based neonatal care by community health workers for preventing mortality in neonates in low- and middle-income countries: a systematic review. J Perinatol [Internet]. 2016 [cited 2019 Mar 
18];36 Suppl 1(Suppl 1):S55-S73. Available from: http://www.ncbi.nlm.nih. gov/pubmed/27109093.

26. Dawson AJ, Buchan J, Duffield C, Homer CSE, Wijewardena K. Task shifting and sharing in maternal and reproductive health in low-income countries: a narrative synthesis of current evidence. Health Policy Plan [Internet]. 2014 May 1 [cited 2019 Mar 18];29(3):396-408. Available from: https://www.ncbi. nlm.nih.gov/pubmed/23656700.

27. Tso LS, Best J, Beanland R, Doherty M, Lackey M, Ma Q, et al. Facilitators and barriers in HIV linkage to care interventions: a qualitative evidence review. AIDS [Internet]. 2016 [cited 2019 Mar 18];30(10):1639-1653. Available from: http://www.ncbi.nlm.nih.gov/pubmed/27058350.

28. Hirschhorn LR, Baynes C, Sherr K, Chintu N, Awoonor-Williams JK, Finnegan K, Philips JF, Anatole M, Bawah AA, Basinga P. Approaches to ensuring and improving quality in the context of health system strengthening: a crosssite analysis of the five African Health Initiative Partnership programs. BMC health services research. 2013;13(2):S8.

29. Manzi A, Magge H, Hedt-Gauthier BL, Michaelis AP, Cyamatare FR, Nyirazinyoye L, Hirschhorn LR, Ntaganira J. Clinical mentorship to improve pediatric quality of care at the health centers in rural Rwanda: a qualitative study of perceptions and acceptability of health care workers. BMC health services research. 2014;14(1):275.

30. Magge H, Anatole M, Cyamatare FR, Mezzacappa C, Nkikabahizi F, Niyonzima $\mathrm{S}$, et al. Mentoring and quality improvement strengthen integrated management of childhood illness implementation in rural Rwanda. Arch Dis Child. 2015;100(6):565-70.

31. Zulu JM, Kinsman J, Michelo C, Hurtig A-K. Integrating national communitybased health worker programmes into health systems: a systematic review identifying lessons learned from low-and middle-income countries. BMC Public Health [Internet]. 2014 Dec 22 [cited 2019 Mar 18];14(1):987. Available from: https://www.ncbi.nlm.nih.gov/pmc/articles/PMC4192351/.

32. Noordam AC, Carvajal-Velez L, Sharkey AB, Young M, Cals JWL. Care seeking behaviour for children with suspected pneumonia in countries in subSaharan Africa with high pneumonia mortality. Patra J, editor. PLoS One. 2015 Feb;10(2):e0117919.

33. Sunguya $B F$, Mlunde $L B$, Ayer $R$, Jimba $M$. Towards eliminating malaria in high endemic countries: the roles of community health workers and related cadres and their challenges in integrated community case management for malaria: a systematic review. Malar J [Internet]. 2017 [cited 2019 Mar 18];16: 10. Available from: https:/www.ncbi.nlm.nih.gov/pmc/articles/PMC5209914/.

34. Proctor EK, Powell BJ, McMillen JC. Implementation strategies: recommendations for specifying and reporting. Implement Sci [Internet]. 2013 Dec 1 [cited 2019 Jun 14];8(1):139. Available from: https://www.ncbi. nlm.nih.gov/pmc/articles/PMC3882890/.

35. Curran GM, Bauer M, Mittman B, Pyne JM, Stetler C. Effectivenessimplementation hybrid designs: combining elements of clinical effectiveness and implementation research to enhance public health impact. Med Care [Internet]. 2012 Mar [cited 2018 Oct 30];50(3):217-226. Available from: http://www.ncbi.nlm.nih.gov/pubmed/22310560

36. Leeman J, Birken SA, Powell BJ, Rohweder C, Shea CM. Beyond "implementation strategies": classifying the full range of strategies used in implementation science and practice. Implement Sci [Internet]. 2017 Dec 3 [cited 2019 Jun 16];12(1):125. Available from: https://www.ncbi.nlm.nih.gov/ pmc/articles/PMC5670723/.

37. Bernet AC, Willens DE, Bauer MS. Effectiveness-implementation hybrid designs: implications for quality improvement science. Bernet al Implement Sci [Internet]. 2012 [cited 2018 Oct 30];(8):2. Available from: https:/www. ncbi.nlm.nih.gov/pmc/articles/PMC3633015/.

38. Fetters MD, Curry LA, Creswell JW. Achieving integration in mixed methods designs_-principles and practices. Health Serv Res [Internet]. 2013 [cited 2019 May 15];48(6 Pt 2):2134. Available from: https://www.ncbi.nlm.nih.gov/ pmc/articles/PMC4097839/.

39. Langlois E V, Montekio VB, Young T, Song K, Alcalde-Rabanal J, Tran N. Enhancing evidence informed policymaking in complex health systems: lessons from multi-site collaborative approaches. 2016 [cited 2019 Mar 11]; Available from: https:/www.ncbi.nlm.nih.gov/pmc/articles/PMC4794922/.

40. Wolfenden L, Williams CM, Wiggers J, Nathan N, Yoong SL. Improving the translation of health promotion interventions using effectivenessimplementation hybrid designs in program evaluations. Heal Promot J Aust [Internet]. 2016 Dec 1 [cited 2019 Jan 17];27(3):204-207. Available from: https://www.ncbi.nlm.nih.gov/pubmed/29241482.
41. World Health Organization. Service Availability and Readiness Assessment (SARA): an annual monitoring system for service delivery, version 2.1. Geneva; 2013.

42. Glasgow RE, Vogt TM, Boles SM. Evaluating the public health impact of health promotion interventions: the RE-AIM framework. Am J Public Health. 1999;89(9):1322-7.

43. Glasgow RE, Estabrooks PE. Pragmatic applications of RE-AlM for health care initiatives in community and clinical settings. Prev Chronic Dis [Internet]. 2018 Jan 4 [cited 2019 Apr 11];15:170271. Available from: https://www.ncbi. nlm.nih.gov/pmc/articles/PMC5757385/.

44. Glasgow RE, Klesges LM, Dzewaltowski DA, Estabrooks PA, Vogt TM Evaluating the impact of health promotion programs: using the RE-AIM framework to form summary measures for decision making involving complex issues. Health Educ Res. 2006;21(5):688-94.

45. Steckler A, McLeroy KR. The importance of external validity. Am J Public Health [Internet]. 2009 [cited 2019 Jan 31];98(1):9-10. Available from: https:// www.ncbi.nlm.nih.gov/pmc/articles/PMC2156062/pdf/0980009.pdf.

46. Mackenzie M, O'Donnell C, Halliday E, Sridharan S, Platt S. Do health improvement programmes fit with MRC guidance on evaluating complex interventions? BMJ [Internet]. 2010 Feb 1 [cited 2019 Jan 22];340:c185. Available from: http://www.ncbi.nlm.nih.gov/pubmed/20123834.

47. Ghaffar A, Langlois E V, Rasanathan K, Peterson S, Adedokun L, Tran NT. Strengthening health systems through embedded research. Bull World Health Organ [Internet]. 2017 [cited 2019 Jan 22];95(2):87. Available from: http://www.ncbi.n/m.nih.gov/pubmed/28250505.

48. Collins D, Jarrah Z. Community health planning and costing tool [Internet]. Management Sciences for Health; 2016 [cited 2018 Oct 22]. Available from: https://www.msh.org/resources/community-health-planning-and-costingtool.

49. Walker N, Friberg IK. Introduction: reporting on updates in the scientific basis for the Lives Saved Tool (LiST). BMC Public Health [Internet]. 2017 Nov 7 [cited 2018 Oct 22];17(S4):774. Available from: https://www.ncbi.nlm.nih. gov/pmc/articles/PMC5688438/.

50. Hemming K, Haines TP, Chilton PJ, Girling AJ, Lilford RJ. The stepped wedge cluster randomised trial: rationale, design, analysis, and reporting. BMJ. 2015 Feb;350:h391.

51. Hayes RJ, Moulton LH. Cluster randomised trials. Boca Raton: Taylor \& Francis Group, LLC; 2009

52. Hemming K, Taljaard M, Grimshaw J. Introducing the new CONSORT extension for stepped-wedge cluster randomised trials. Trials [Internet]. 2019 Dec 18 [cited 2019 Jan 25];20(1):68. Available from: https://trialsjournal. biomedcentral.com/articles/10.1186/s13063-018-3116-3.

53. Ministre de la Sante et de la Protection Sociale (MSPS). Plan National de Developpement Sanitaire 2017-2022. Lomé, Togo; 2017.

54. Kish L. A Procedure for objective respondent selection within the household. J Am Stat Assoc. 1949:44(247):380-7.

55. Baio G, Copas A, Ambler G, Hargreaves J, Beard E, Omar RZ. Sample size calculation for a stepped wedge trial. Trials Baio al Trials. 2015;16(16).

56. Belza BA, Toobert DJ, Glasgow RE. RE-AIM for program planning: Overview and applications. Washington, DC: National Council on Aging. 2007;165.

57. Graham W, Brass W, Snow RW. Estimating maternal mortality: the sisterhood method. Stud Fam Plann [Internet]. 1989 May [cited 2018 Nov 7];20(3):125. Available from: https://www.ncbi.nlm.nih.gov/pubmed/2734809.

58. World Health Organization, Division of Reproductive Health. The sisterhood method for estimating maternal mortality: guidance notes for potential users. Geneva; 1997

59. Damschroder LJ. Fostering implementation of health services research findings into practice: a consolidated framework for advancing implementation science. Implement Sci [Internet]. 2009;1-15. https://doi. org/10.1186/1748-5908-4-50.

\section{Publisher's Note}

Springer Nature remains neutral with regard to jurisdictional claims in published maps and institutional affiliations. 